\title{
High sensitivity optoelectronic sensor for nitric oxide detection in exhaled air
}

\author{
J. Wojtas ${ }^{1}$, Z. Bielecki ${ }^{1}$, T. Stacewicz ${ }^{2}$, J. Mikolajczyk ${ }^{1}$ \\ ${ }^{1}$ Institute of Optoelectronics, Military Univ. of Technology, 2 Kaliskiego Str., 00-908 Warsaw, Poland; \\ zbielecki@wat.edu.pl \\ ${ }^{2}$ Institute of Experimental Physics, University of Warsaw, 69 Hoza Str., 00-068 Warsaw, Poland.
}

\begin{abstract}
:
Certain gases in human breath are known to be indicators of the presence of diseases. These gases have been identified as biomarkers using different instruments, mainly based on chemical methods, e.g. gas chromatography or chemiluminescence. The article describes an application one of the most sensitive optoelectronic method - Cavity Enhanced Absorption Spectroscopy (CEAS) to investigation of fractional exhaled nitric oxide (FeNO). Measurement of FeNO concentration is a quantitative, non-invasive, simple, and safe method of respiratory inflammation and asthma diagnosis. For detection of NO the vibronic molecular transitions were used in our optoelectronic sensor. The wavelengths of these transitions are situated in the spectral range of $5.23 \mu \mathrm{m}-5.29 \mu \mathrm{m}$. A setup of the sensor consists of a pulsed quantum cascade laser (QCL), optical cavity, high sensitivity photoreceiver and a digital signal processing system. The optical cavity was built of spherical mirrors of high reflectance. The leakage optical signal from the cavity output was detected with specially developed low noise detection module. In the sensor, the detection limit of $75 \mathrm{ppb}$ (parts per billion) was obtained.
\end{abstract}

Key words: laser absorption spectroscopy, NOx detection, cavity ring down spectroscopy, QC lasers.

\section{Introduction}

Nowadays, many techniques of laser spectroscopy are applied to NO detection. Their detection limit is varied from ppt-level to hundreds of ppm [1-5]. One of the most sensitive methods is cavity ring down spectroscopy (CRDS). The idea of the CRDS is shown in Fig. 1. During the detection procedure, a light pulse is injected into the cavity through one of the mirrors. Inside the cavity multiple reflections are observed. After each reflection, leakage radiation from the cavity is registered with the photodetector.

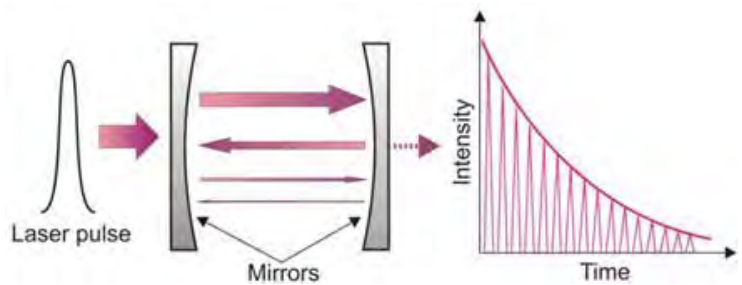

Fig. 1. Cavity ring down spectroscopy idea.

In the cavity, two concave mirrors with a very high reflectivity $R$ are mounted. This construction provides a long optical path, even up to several kilometers [6]. Intensity of radiation that is passing through the tested gas, can be determined using Lambert-Beer-Burger law

$$
\mathrm{I}(\mathrm{t})=\mathrm{I}_{0} \mathrm{e}^{-\frac{[(1-\mathrm{R})+\alpha \mathrm{L}] \mathrm{c}}{\mathrm{L}} \mathrm{t}},
$$

where $I_{0}$ denotes the intensity of injected optical radiation, $\alpha$ is the absorption coefficient, $c$ is the light speed, $L$-optical path, $t$ - time. The cavity losses are inversely proportional to the decay time $\tau$ of exponential decreasing radiation in the cavity. The maximum value of radiation intensity in the cavity is directly proportional to $T$. It is shown that the time-integrated intensity of light leaked out the cavity is linearly proportional to the decay time. Thus by measuring this time parameter, the determination of an absorption coefficient is possible $[7,8]$

$$
T=\frac{L}{c(1-R+\alpha L)}
$$

Thanks to two decay times measurements, in the case of lack of tested gas $\left(\tau_{0}\right)$, and when the cavity is filled with tested gas $(T)$, the absorption coefficient can be determined from the formula 


$$
\alpha=\frac{1}{C}\left(\frac{1}{T}-\frac{1}{T_{0}}\right)
$$

\section{CEAS sensor for NO detection in human breath}

The analysis shown, that application of CEAS method in the IR wavelength range provides possibility to develop NO sensor, the sensitivity of which could reach the ppb level. At the wavelength ranges of $5.23 \mu \mathrm{m}-5.29 \mu \mathrm{m}$, the value of absorption cross section is about
$0.7 \times 10-18 \mathrm{~cm}^{2}$. Additionally, there are no significant interferences of absorption lines of the atmosphere gases like $\mathrm{CO}$ or $\mathrm{O} 2$. There could be observed influence of $\mathrm{H} 2 \mathrm{O}$, which can be minimized with use of special particles filter or dryer, and pressure reduction. NO absorption spectrum is presented in Fig. 2. To assure high sensitivity of the sensor, wavelength of the QC laser radiation is matched to the selected absorption line e.g. $5.2629 \mu \mathrm{m}$.

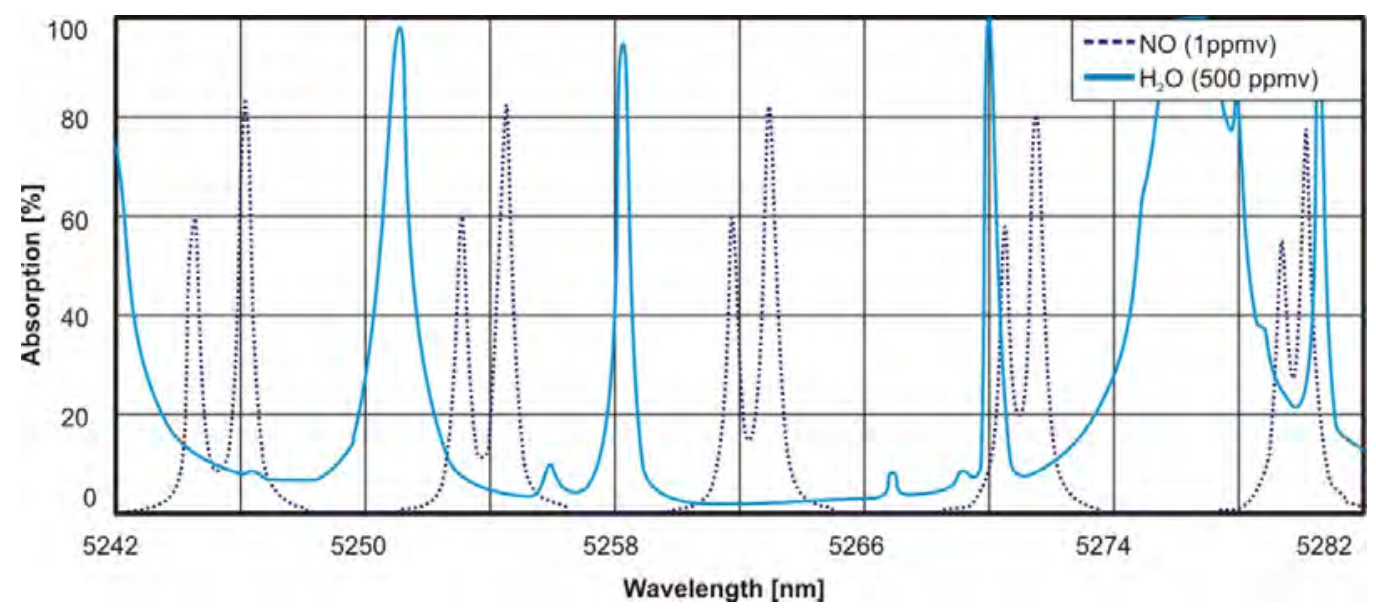

Fig. 2. NO absorption spectrum.

A cavity enhanced absorption spectroscopy is a modification of the CRDS. This method was proposed in 1998 by Engeln et al. [9]. The operation idea of the CEAS technique is shown in Fig. 3.

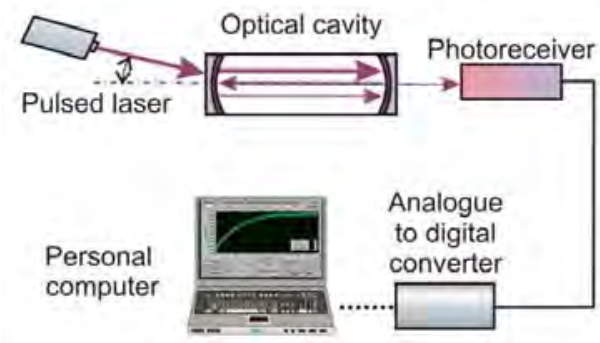

Fig. 3. Idea of CEAS technique.

In the sensor, the laser beam is injected into optical cavity under a very small angle in respect to its axis. It results in the formation of a dense structure of weak modes, which can overlap each other. Sometimes, in addition to the output mirror, a piezoelectric-driven mount is used to modulate the cavity length. Thanks to this, the establishment of a constant mode structure within the cavity is minimized [10]. In the cavity a pair of high-reflective mirrors (Los Gatos Research, Inc.) was used. Their reflectivities reach the value of about $99.98 \%$ at the wavelengths of interest. The distance between them was about $60 \mathrm{~cm}$.

The project of the NO sensor is presented in Fig. 4. It is built of laser control system, optical system, sample module and signal processing system. The main task of the laser control system is to stabilize parameters of applied optical radiation. In the sensor, a quantum cascade laser was applied. Its emission line is very narrow and also is characterized by both high power and good spectral stability. The laser spectra could be tuned with temperature or current changes. Thus, it is necessary to precise control of their operation conditions (voltage, current, and temperature). It should be noticed that the QC laser is very sensitive to electrical surges and instabilities. That is why in the system, high quality power supply was applied (E3634A, Agilent). For pulsed mode of the laser operations, pulse generator (DG645 type, Stanford Research Systems, Inc.) connected to laser driver (LDD400, Alpes Lasers) was used. 


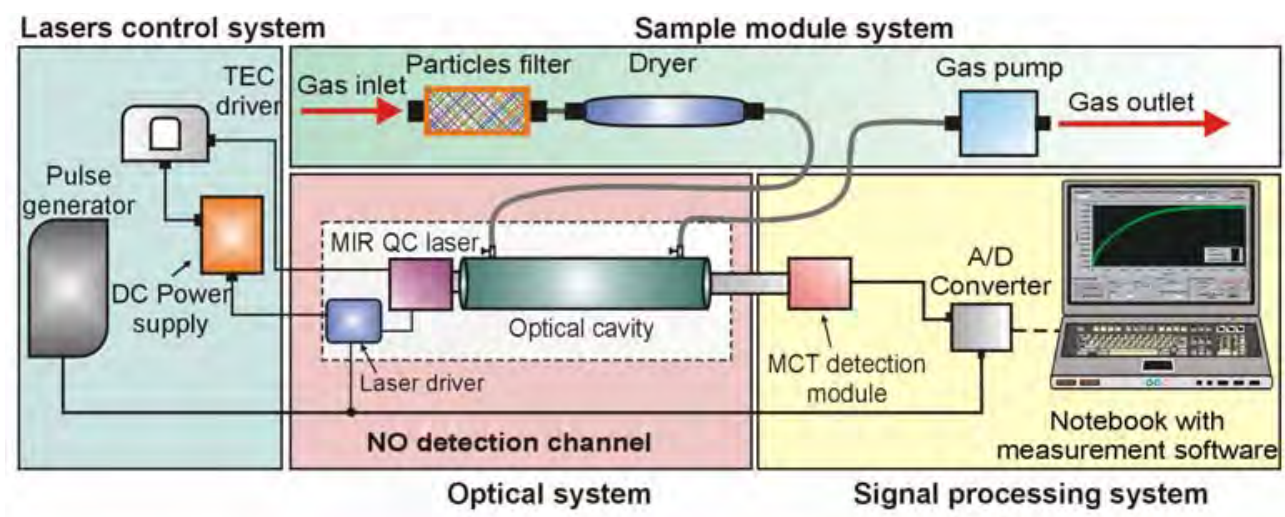

Fig. 4. Block diagram of NO sensor.

Signal from the cavity was registered with detection module - PVI-2TE (VIGO System S.A.). The main element of the module are $\mathrm{HgCdTe}$ (MCT) photodiode and low-noise transimpedance preamplifier [11,12]. For temperature control of the photodiode, twostage thermoelectric cooler was applied. Next, signal from the preamplifier was digitized using $A / D$ converter. The measurements data were transferred via USB interface to the portable computer equipped with special sensor software. The software provides determination of NO concentrations.

During the investigation of the sensor, concentration measurements of reference gas samples were carried out. Gas samples were prepared using the gas standards generator 491M type from Kin-Tek. The modular construction of the generator enables production of multicomponent gas mixtures using different mixing techniques. High precision of mixing ratios of the components from a level of part per trillion (ppt) to the initial concentration of $1: 1$ can be achieved. The generator can produce both dry and moistened standard gas, which can be supplied to the sensor at an adjustable pressure.

In the sensor software, a special algorithm for determination of $\mathrm{NO}$ concentration has been implemented. The measurement was carried out during two-step process that was above described. Knowing the absorption cross section $\sigma$ of the gas, its concentration is calculated from the formula

$$
\mathrm{N}_{\mathrm{x}}=\frac{1}{\mathrm{~N}_{\mathrm{O}} \mathrm{c \sigma}}\left(\frac{1}{\mathrm{~T}}-\frac{1}{\mathrm{~T}_{0}}\right)
$$

where $N_{o}$ denotes the Loschmidt's number. The panel of the software is shown in Fig. 5. a)

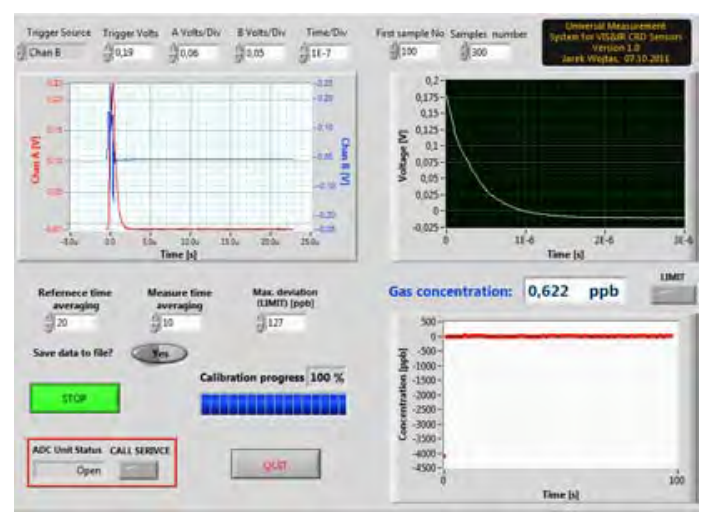

b)

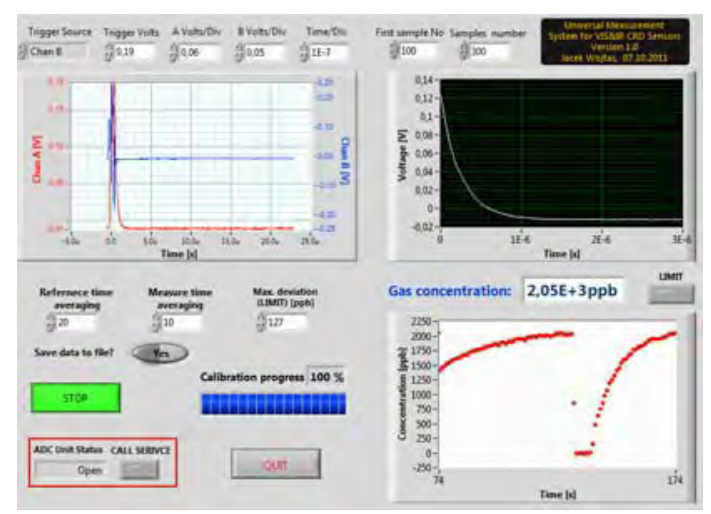

Fig. 5. The view of software panel during a flow of inert gas - argon (a) and during the measurement of nitric oxide concentration (b).

Figure 6 presents the results of $\mathrm{NO}$ concentration measurements of the reference samples. Maximum uncertainty of nitric oxide determination was not higher than $12 \%$. 


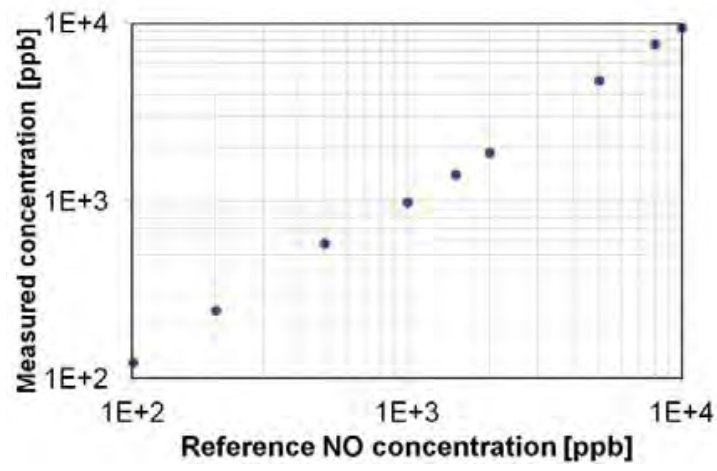

Fig. 6. Results of concentration measurements of NO reference samples.

\section{Summary}

In the paper the cavity enhanced absorption spectroscopy technique applied to nitric oxide sensor was presented. The constructed sensor is able to measure NO concentration at $\mathrm{ppb}$ level. Its sensitivity is comparable with sensitivities of instruments basing on other methods, e.g. gas chromatography or mass spectrometry. However its selectivity is much higher. The developed sensor can be applied to monitoring of atmosphere quality and asthma diagnosis.

\section{Acknowledgements}

The presented works were supported by The National Centre for Research and Development and National Science Centre in the scope of projects: No. OR00002807, ID: 151673 and ID: 179900.

\section{References}

[1] Sigrist, M.W., Air monitoring by spectroscopic techniques, John Wiley \& Sons, New York, USA, (1994).

[2] Li, P.; Shi, K.; Liu, Z., Optical scattering spectroscopy by using tightly focused supercontinuum. Opt. Express, 13, no. 22, 90399044, (2005).

[3] Lagalante, A.F., Atomic Absorption Spectroscopy: A Tutorial Review. Applied Spectroscopy Reviews, 34(3), pp.173-189, (1999).

[4] O'Keefe, A., Integrated cavity output analysis of ultra-weak absorption, Chemical Physics Letters , 293 (5), pp. 331-336, (1998).

[5] Noel, S.; Bovensmann, H.; Burrows, J.P.; Frerick, J.; Chance', K.V.; Goede, A.H.P., Global Atmospheric Monitoring with SCIAMACHY. Physal. Chemical. Earth, 24, no. 5, pp. 427434, (1999).

[6] Busch, K.W.; Busch, M.A., Cavity-Ringdown Spectroscopy. ACS Symposium series, American Chemical Society, Washington DC, USA, (1999).
[7] Wojtas, J.; Czyzewski, A.; Stacewicz, T.; Bielecki, Z., Sensitive detection of NO2 with Cavity Enhanced Spectroscopy, Optica Applicata, 36(4), pp. 461-467, (2006).

[8] Wojtas, J.; Bielecki, Z., Signal processing system in the cavity enhanced spectroscopy, OptoElectron. Rev., 16(4), pp. 44-51, (2008).

[9] Engeln, R.; Berden, G.; Peeters, R.; Meijer, G., Cavity enhanced absorption and cavity enhanced magnetic rotation spectroscopy, Review of Scientific Instruments, 69 , no. 11, pp. 3763 3769, (1998).

[10] Paul, J.B.; Lapson, L.; Anderson, J.G., Ultrasensitive absorption spectroscopy with a high-finesse optical cavity and off-axis alignment. App. Opt., Vol. 40, No. 27, pp. 49044910, (2001).

[11] Piotrowski, A.; Madejczyk, P.; Gawron, W.; Klos, K.; Romanis, M.; Grudzien, M.; Rogalski, A.; Piotrowski, J., MOCVD growth of $\mathrm{Hg} 1 \times \mathrm{CdxTe}$ heterostructures for uncooled infrared photodetectors, Opto-Electron. Rev., 12, pp. 453-458, (2004).

[12] Rogalski, A.; Bielecki, Z., Detection of optical radiation (chapter A1.4), Handbook of optoelectronics, Taylor \& Francis, New York, (2006). 